\title{
BOOKS
}

\section{Second volume of infectious diseases series}

Handbook of Infectious Diseases: Obstetric and Perinatal Infection, edited by David Charles (1993). Mosby Year Book, Inc, 11830 Westline Industrial Drive, St Louis, Missouri 63146, USA. ISBN 1-55664-395-0, 365 pages.

This is the second book in the Handbook of Infectious Diseases series, a conception of the late Edward Kass. This text was originally published two decades ago; that first edition was co-edited by David Charles and Maxwell Finland. The goals for the current edition are to provide "a rich source of current knowledge regarding obstetric and perinatal infections" and a "trustworthy foundation for further progress". Twenty-seven chapters are incorporated into two large sections: the mother, the mother and fetus; and a third smaller section, the neonate. These chapters cover a wide spectrum of infectious syndromes and specific etiological agents relevant to pregnancy and the puerperium.

The audience for this book would primarily be the obstetrics and gynecology practitioner who requires in-depth clinical information for selected infections. Generally speaking, microbiological, pathogenetic, immunological and other basic information is dealt with superficially, with the emphasis on differential diagnosis, clinical assessment and therapy. There are, however, some exceptions. Discussions of basic aspects of vaginal flora, viral infections, AIDS, toxoplasmosis, group B streptococcal disease, listeriosis, syphilis and postpartum infections provide more basic details. The clinical information is generally complete, although distressingly anecdotal in some chapters.

Some serious factual errors in the text, especially in therapeutic aspects, are of concern. The chapter entitled The use and abuse of antibiotics in obstetric practice' suggests that quinolones should be used for the treatment of urinary tract infection in pregnancy, that clindamycin is appropriate therapy for Listeria monocytogenes, and that Staphylococcus aureus endocarditis should be preferentially treated with a cephalosporin. These and some other comments are erroneous or misleading. In discussing urinary tract infections, the recommended treatment for asymptomatic bacteriuria is nitrofurantoin $100 \mathrm{mg}$ at bedtime for 10 days - an unusual dosage duration and regimen unsupported by pharmacokinetic data or clinical studies. The discussion of anti-infective chemotherapy and obstetrics and gynecology becomes confusing and even contradictory as, for instance, rifampin is stated to be a category $\mathrm{B}$ drug on one page and a category $\mathrm{C}$ drug with respect to pregnancy on the next page.

The chapter discussing 'The epidemiology of obstetric infections' is interesting and could have been a well reasoned plea for more careful attention to traditional surgical and infection control techniques in infection prevention. Unfortunately it is more a diatribe providing simplistic approaches to complex problems. It does not provide a convincing appraisal of available data to buttress arguments for the importance of surgical technique in asepsis and infection prevention.

Some information is dated or incomplete. The terminology for viral hepatitis is not current and, oddly, while this chapter discusses viral hepatitis it has limited discussion of hepatitis in pregnancy. The discussion of tuberculosis is, again, superficial and does not address issues related to human immunodeficiency virus infection and resistance. It too seems lacking in directly addressing issues of tuberculosis specific to pregnancy, particular with respect to potential increased risks for reactivation or progression. A discussion of antibiotic concentrations in breast milk is limited to beta-lactam antibiotics only. The discussion of septic shock does not discuss the role of cytokines nor does it discourage use of steroids. While there are a few sentences mentioning ophthalmia neonatorum in chapters describing specific etiological agents, no complete discussion of this important problem is found in the text.

In summary, this book has some excellent individual chapters and may be of value, particularly for clinical issues for practitioners whose primary field is obstetrics and gynecology. It is, generally, too superficial to be uniformly valuable for individuals whose background is microbiology or infectious diseases. As noted, some errors in content limit its potential utility, particularly with respect to antimicrobial use.

LE Nicolle, MD Winnipeg. Maintoba 


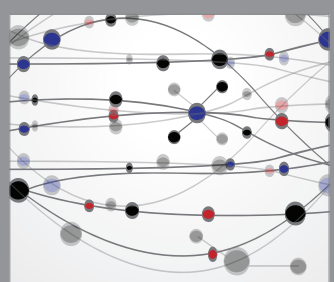

The Scientific World Journal
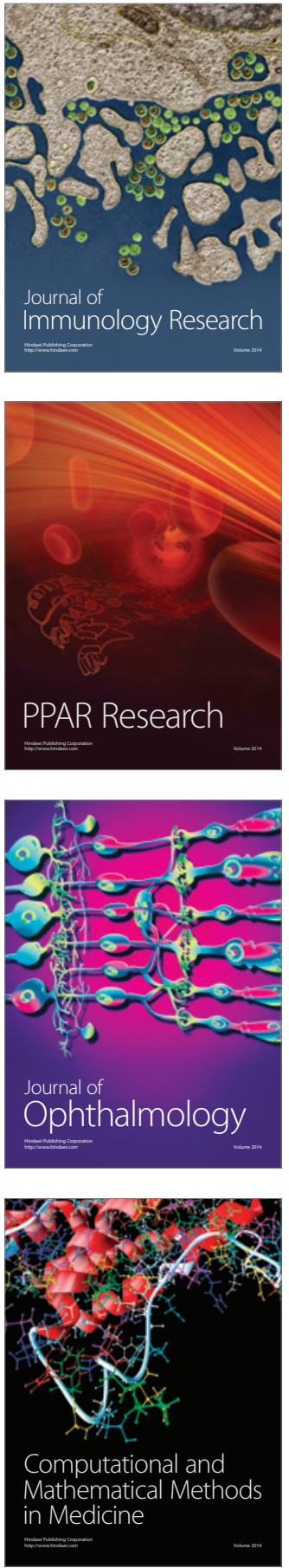

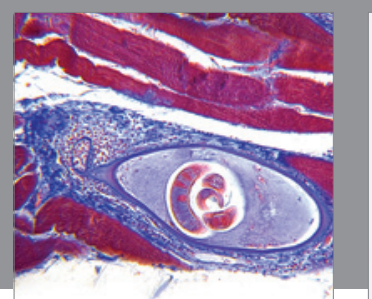

Gastroenterology Research and Practice

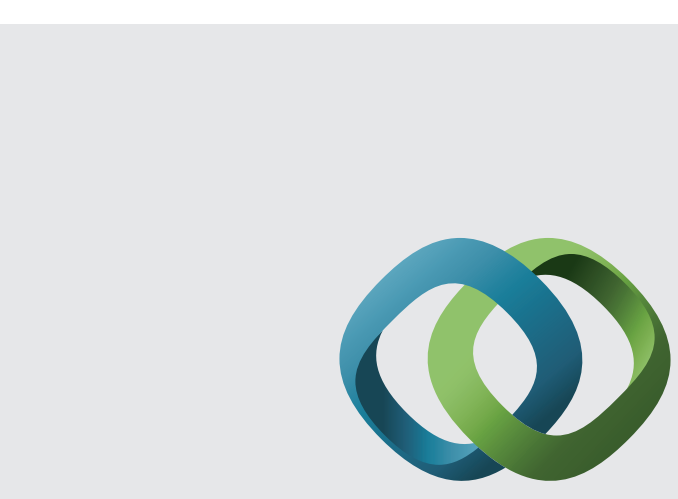

\section{Hindawi}

Submit your manuscripts at

http://www.hindawi.com
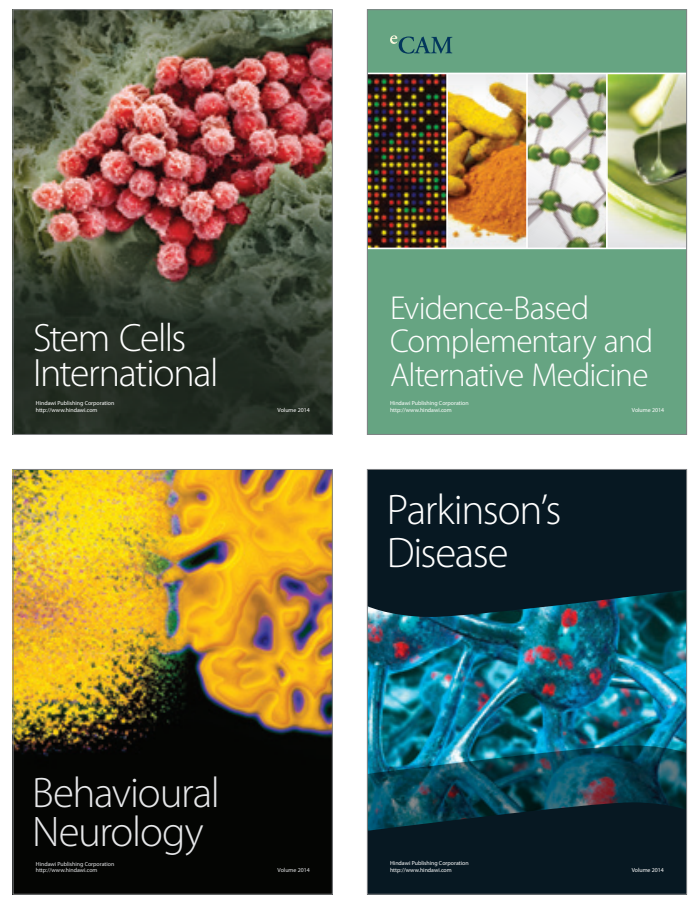
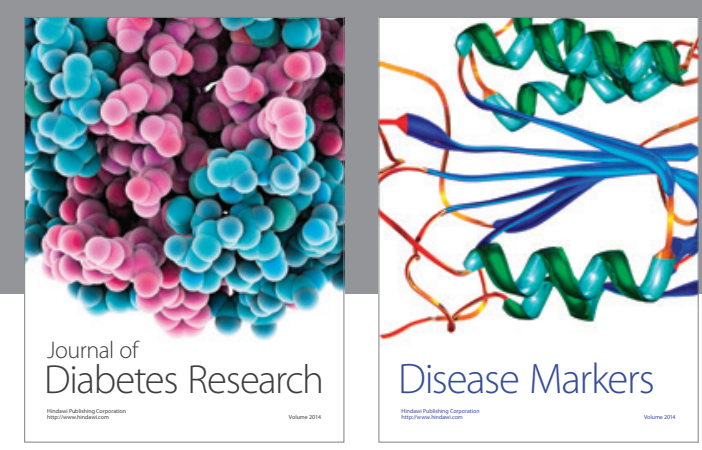

Disease Markers
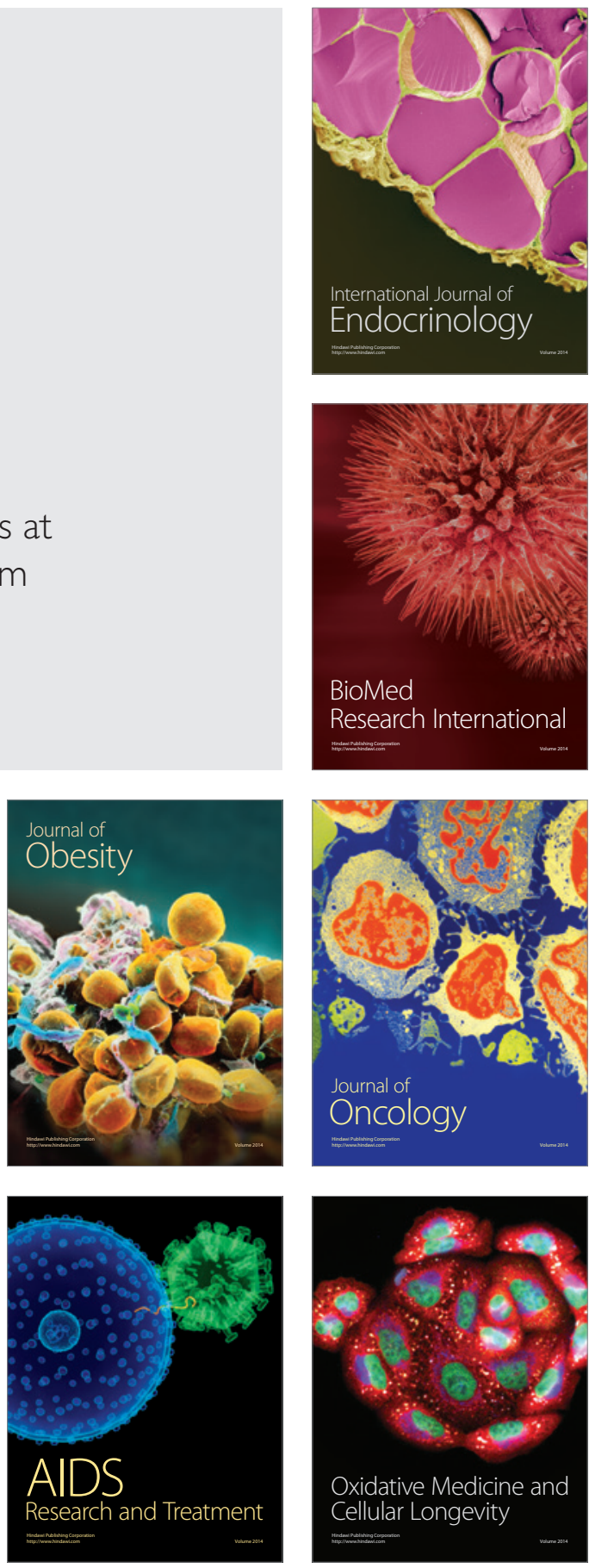